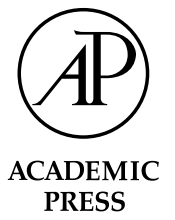

\title{
Impaired processing of prosodic and musical patterns after right hemisphere damage
}

\author{
Karen G. Nicholson, ${ }^{\mathrm{a}, \mathrm{b}, *}$ Shari Baum, ${ }^{\mathrm{c}}$ Andrea Kilgour, ${ }^{\mathrm{a}}$ Christine K. Koh, ${ }^{\mathrm{a}}$ \\ K.G. Munhall, ${ }^{\mathrm{a}, \mathrm{b}, \mathrm{d}}$ and Lola L. Cuddy ${ }^{\mathrm{a}}$ \\ a Department of Psychology, Queen's University, Kingston, Ontario, Canada \\ ${ }^{\mathrm{b}}$ Department of Otolaryngology, Queen's University, Kingston, Canada \\ ${ }^{\mathrm{c}}$ School of Communication Sciences and Disorders, McGill University, Montreal, Canada \\ d ATR Human Information Science Laboratories, Kyoto, Japan
}

Accepted 23 June 2003

\begin{abstract}
The distinction between the processing of musical information and segmental speech information (i.e., consonants and vowels) has been much explored. In contrast, the relationship between the processing of music and prosodic speech information (e.g., intonation) has been largely ignored. We report an assessment of prosodic perception for an amateur musician, KB, who became amusic following a right-hemisphere stroke. Relative to matched controls, KB's segmental speech perception was preserved. However, KB was unable to discriminate pitch or rhythm patterns in linguistic or musical stimuli. He was also impaired on prosodic perception tasks (e.g., discriminating statements from questions). Results are discussed in terms of common neural mechanisms that may underlie the processing of some aspects of both music and speech prosody.
\end{abstract}

(C) 2003 Elsevier Inc. All rights reserved.

\section{Introduction}

Past research on auditory processing at the cortical level has been closely related to the processing of speech and language. More recently, however, there has been an increased interest in both the processing of nonspeech auditory stimuli (e.g., music, emotion, and talker identity) and the extent to which it overlaps with the processing of speech (for reviews see Griffiths, 2002; Polster \& Rose, 1998). The focus of the present investigation is the relationship between the processing of musical information and prosodic speech information.

Segmental speech information (i.e., consonants and vowels) and musical information are conveyed by markedly different changes in the acoustic signal (for review see Zatorre, Belin, \& Penhune, 2002). Segmental speech information is conveyed by rapid changes in

\footnotetext{
${ }^{*}$ Corresponding author. Current address: Department of Psychology, Crabtree Bldg., 49A York St., Mount Allison University, Sackville, New Brunswick E4L 1C7, Canada. Fax: +506-364-2467.

E-mail address: knicholson@mta.ca (K.G. Nicholson).
}

acoustic events (e.g., formant transitions), while musical information is conveyed by slower, structured variations in fundamental frequency $\left(F_{0}\right)$, duration, and intensity. In contrast, the perception of prosodic speech information (i.e., sentence intonation, rhythm, and stress) and the perception of musical information rely on similar changes in the acoustic signal (e.g., Bolinger, 1989). For instance, both speech intonation and melodic contour rely on variations in $F_{0}$ over time. Also, the temporal grouping of information in both speech and music appears to rely on the same durational cues, such as a slowing before the phrase boundary (see for review Patel, Peretz, Tramo, \& Labreque, 1998).

The dominant view is that music perception is mediated by different neural mechanisms than speech perception. Support for this view comes largely from reports of patients who show selective auditory agnosias (e.g., Ayotte, Peretz, Rousseau, Bard, \& Bojanowski, 2000; Dalla Bella \& Peretz, 1999; Peretz et al., 1994). More specifically, patients have been identified who are impaired at perceiving music, but are still able to perceive speech and environmental sounds. Conversely, 
other patients have been reported who are impaired at perceiving speech, but have a preserved ability to perceive music and environmental sounds. Although this dissociation in the neural mechanisms mediating the perception of music and segmental speech information (i.e., consonants and vowels) has been well established, the relationship between the neural mechanisms mediating the perception of music and prosodic speech information has been largely ignored (but see Besson \& Schon, 2001; Zatorre, 2001).

Patel and colleagues (1998) examined the ability of two patients, who presented with amusia subsequent to bilateral brain damage, to process pitch and rhythm patterns in linguistic and musical stimuli. The first patient (the late $\mathrm{CN}$ ) was an "associative" amusic. $\mathrm{CN}$ was impaired at recognizing familiar music, although she was able to discriminate pitch and rhythm patterns. Her deficit was attributed to a problem in accessing stored melodic representations, rather than a problem perceiving acoustic patterns (Peretz, 1996). Consistent with this argument, $\mathrm{CN}$ performed at control levels on tasks requiring a same-different discrimination of two sequentially presented sentences based on pitch or rhythm patterns. She was also able to discriminate two sequentially presented musical versions of the sentences, using pitch or rhythm patterns (i.e., these were derived by replacing the syllables with tones, while maintaining pitch and timing information). In addition, $\mathrm{CN}$ was able to perform tasks that required the extraction of prosodic information from a sentence, tasks such as deciding whether sentences were statements or questions or deciding which word carried the emphasis in a sentence.

In contrast, the second patient (IR) is an "apperceptive" amusic. Like CN, IR is impaired at recognizing familiar music, but, unlike $\mathrm{CN}$, she is impaired at discriminating pitch and rhythm patterns. IR's deficit has been attributed to an inability to process acoustic patterns underlying music perception, rather than an inability to access stored melodic representations. IR was impaired at discriminating sentences or musical stimuli derived from these sentences, based on pitch or rhythm pattern. Despite her deficit on prosodic discrimination tasks IR was not impaired at extracting prosodic information from a sentence, to decide whether a sentence was a statement or question or to decide which word in a sentence carried the emphasis.

Patel and colleagues concluded that some aspects of music and speech prosody might rely on a shared neural substrate. To elucidate potential substrates, they contrasted the pattern of damage in $\mathrm{CN}$ and IR, both of whom had extensive bilateral damage. IR had damage to the right frontal cortex, whereas $\mathrm{CN}$ did not, suggesting that a neural system involving the right frontal cortex may be involved in processing prosodic and musical information. IR was impaired on prosodic discrimination tasks, but not on tasks requiring the extraction of prosodic in- formation from a sentence (e.g., differentiating statements from questions). Given this, Patel et al. suggested that the right frontal cortex might be involved in the short-term retention and comparison of pitch and temporal patterns, rather than extracting these patterns from the acoustic signal. This notion is consistent with neuroimaging findings showing that the right frontal cortex is implicated in the retention and comparison of pitch in both the speech and music domains (e.g., Zatorre, 2001).

In the present investigation, we examined the ability of another amusic patient (KB) to perform the discrimination tasks and prosodic perception tasks used by Patel and colleagues. KB is severely impaired on basic tests of pitch and rhythm discrimination as well as melody recognition, an indication that, like IR, his musical problems are likely at the perceptual stage (Steinke, Cuddy, \& Jakobson, 2001). KB is not aphasic and he has an intact ability to perceive segmental speech and environmental sounds (Steinke et al., 2001; Nicholson, Baum, Cuddy, \& Munhall, 2002). In spite of these spared auditory abilities, KB is markedly impaired on prosodic speech perception tasks (e.g., differentiating statements from questions) in both the auditory and visual modalities (Nicholson et al., 2002).

It is possible that his deficit in both prosodic and musical processing result from damage to a common neural substrate involved in the perception of acoustic patterns. $\mathrm{KB}$ differs from $\mathrm{CN}$ and IR in that his amusia is consequent to unilateral damage to the right frontoparietal cortex, sparing the temporal cortex. Most cases of amusia, including $\mathrm{CN}$ and IR, result from extensive bilateral damage that typically includes auditory areas in both temporal lobes (Griffiths, 2002). It is notable that the few other cases of amusia reported after unilateral damage resulted from damage to the right parietal cortex (Griffiths et al., 1997).

In summary, KB's auditory deficits appear to be restricted to processing musical and prosodic information. The fact that his brain damage is relatively confined, compared to other cases of amusia, provides an opportunity to examine the question whether common neural substrates might be involved in perceiving music and prosody. In the present investigation we tested KB's ability to perform the discrimination tasks and prosodic perception tasks used by Patel and colleagues. Although we have tested $\mathrm{KB}$ on prosodic perception tasks, we have not administered prosodic discrimination tasks. One aim of the present investigation was to examine whether KB's aprosodia corresponds to his amusia in that it results from a perceptual deficit, rather than a deficit in the knowledge of prosodic categories. A second aim of the this study was to replicate Patel et al.'s finding that the discrimination performance of both amusic patients and matched controls is correlated across the speech and music domains, when the stimuli are equated for pitch and timing patterns. 


\section{Method}

\subsection{Participants}

\subsection{1. $K B$}

A detailed description of KB is given in Steinke et al. (2001). He is a 72 year old, right-handed, native Englishspeaking male with 13 years of formal education, who worked in law enforcement. He has approximately 13 years of music performance experience (vocal and instrumental). At age 64, KB presented with left-sided paralysis and speech production difficulties. His speech problems resolved in a few days, but his paralysis persists. CT scans, taken 6, 8, and 12 months after his stroke, showed focal damage in the frontoparietal area (see Fig. 1), cerebellum, and lenticular nucleus of the right hemisphere. In addition, there was evidence of diffuse brain atrophy that was consistent with KB's age. KB's written consent was obtained prior to testing.

Cognitive functioning. A standard neuropsychological assessment was conducted 7-10 weeks after KB's stroke.
KB's Wechsler Memory Quotient was 109 (Wechsler Memory Scale-Form 1; Stone, Girdner, \& Albrecht, 1946), indicating normal memory function for his age. Tests from the Wechsler Adult Intelligence Scale-Revised (WAIS-R; Wechsler, 1981) revealed a full-scale IQ (FIQ) of 92, a verbal IQ (VIQ) of 103 and a performance IQ (PIQ) of 80. Premorbid estimates of WAIS-R IQ, based on his results on the North American Adult Reading Test (NAART; Blair \& Spreen, 1989), indicated a FIQ of 107, a VIQ of 107, and a PIQ of 108. These results indicate that KB's verbal intellectual functioning was intact post-stroke, while his nonverbal intellectual functioning had declined. This decline in nonverbal cognitive functioning was also indicated by KB's poor performance $(<25$ th percentile) on several tests that are sensitive to parietal and frontal damage. These included the Raven's Coloured Progressive Matrices Test (Raven, 1965), the Trail-Making Test, the Rey-Osterrieth Complex Figure Test (Rey, 1941), the House-Tree-Person Test, and the Wisconsin Card Sorting Test (Heaton, 1981). a

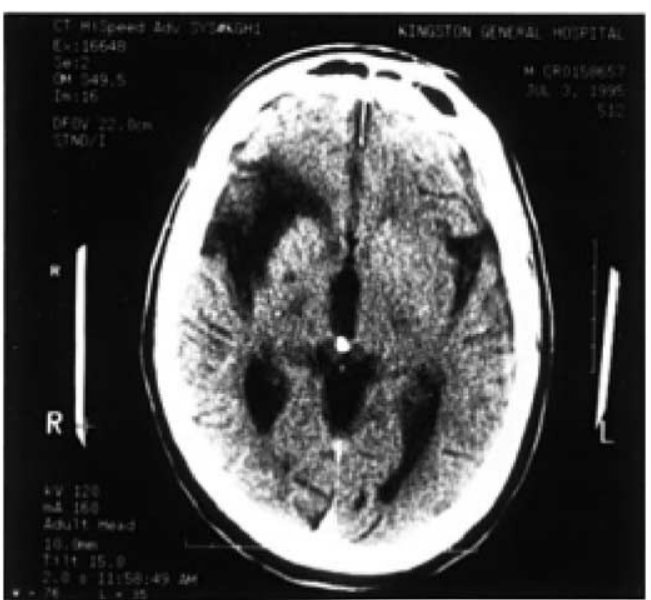

C

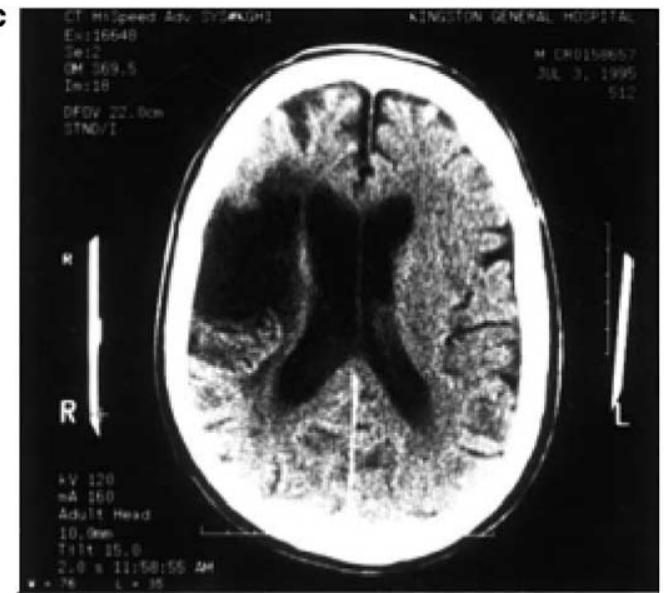

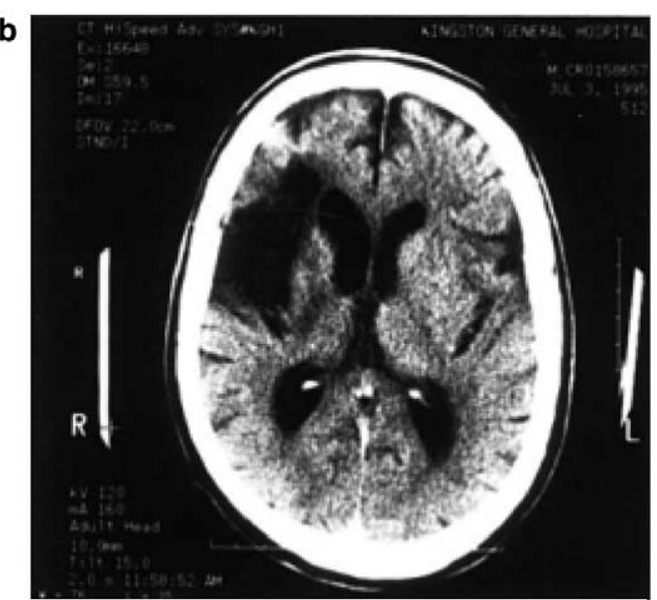

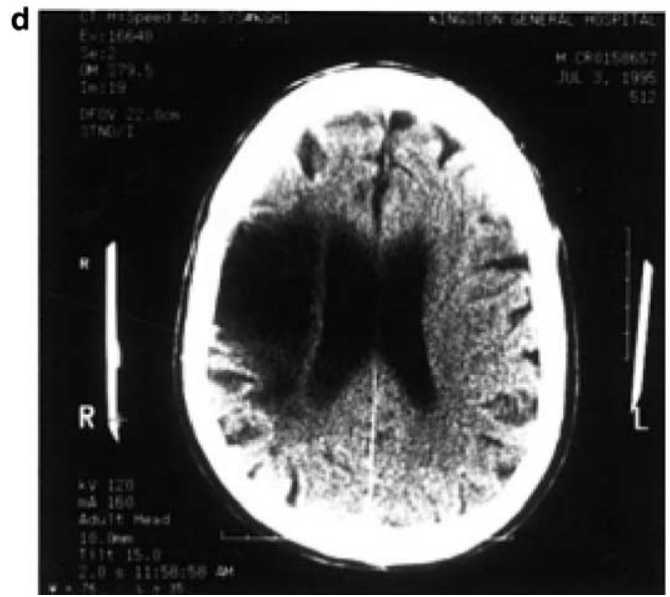

Fig. 1. Transverse CT scans ( $\left(\right.$ ilt $=15^{\circ}$ ) obtained from KB 12 months post-stroke, showing focal damage in the right frontoparietal cortex. The right side of the brain is shown on the left in each image. The four images, labeled (a)-(d), proceed superiorly in 10 mm increments. Reprinted by permission of Psychology Press Ltd., Hove, UK, from Steinke, W.R., Cuddy, L.L., \& Jakobson, L.S. 2001, Dissociations among functional subsystems governing melody recognition after right hemisphere damage. Cognitive Neuropsychology, 18, $411-437$. 
Audiometric testing. Because of KB's frail health, he was not moved to clinical facilities for testing but was tested in a quiet room in his nursing home. As testing conditions were not ideal, however, deviations from normal performance may be over-estimated. A pure tone audiogram (GSI 17 Audiometer, Electro-medical Instrument, Mississauga, Ont.) showed a pattern of hearing loss that was consistent with his age (good ear: $.5 \mathrm{kHz}=25 \mathrm{~dB} \mathrm{HL} ; .75 \mathrm{kHz}=35 \mathrm{~dB} \mathrm{HL} ; 1 \mathrm{kHz}=45 \mathrm{~dB}$ $\mathrm{HL} ; 2 \mathrm{kHz}=50 \mathrm{~dB} \mathrm{HL}$; and $4 \mathrm{kHz}=75 \mathrm{~dB} \mathrm{HL}){ }^{1}$

\subsubsection{Auditory processing. KB presented with a} marked and selective deficit in processing musical stimuli (for scores see Steinke et al., 2001). Administration of the Boston Diagnostic Aphasia Examination (BDAE; Goodglass \& Kaplan, 1983) revealed that KB was not aphasic. In addition, he was able to identify musical instruments by sound, as well as non-speech environmental sounds. In contrast, KB had problems both producing and perceiving melody after his stroke. He was poor at singing and rhythm reproduction, which he and his wife confirmed was not the case pre-stroke. He was impaired at recognizing familiar melodies. ${ }^{2} \mathrm{He}$ had deficits on pitch and rhythm pattern discrimination tasks, suggesting that his deficits were perceptual in origin. Finally, KB was also judged as having a "lack of melody" in his spontaneous speech, which both he and his wife confirmed was not true pre-stroke.

\subsubsection{Control participants}

Twelve individuals (five males, seven females) were recruited from the Kingston community through newspaper advertisements and all gave their written consent prior to testing. These individuals were in good health, had no history of neurological and/or psychiatric disease, and passed an audiometric screening that required them to detect a $.5,1$, and $2 \mathrm{kHz}$ tone at $35 \mathrm{~dB}$ HL. Controls had a mean age of 70 years (range: 67-75

\footnotetext{
${ }^{1}$ Although KB shows some evidence of age-consistent hearing loss at higher frequencies it is unlikely that this is the sole cause of his deficits in processing musical or prosodic information. $\mathrm{KB}$ is still able to process segmental speech information, in spite of the fact that this information is higher in frequency that prosodic speech information. In addition, although we excluded the data from control participants who did not pass a screening test for hearing (i.e., were able to hear audiometric tones at $.5,1$, and $2 \mathrm{kHz}$ at $35 \mathrm{~dB} \mathrm{HL}$ ), one control with bilateral hearing aids performed well above chance on all tasks reported in this paper.

${ }^{2} \mathrm{~KB}$ showed an interesting dissociation in his ability to recognize familiar melodies (Steinke et al., 2001). Although he was impaired at recognizing familiar instrumental melodies (e.g., The Blue Danube), he had a preserved ability to recognize familiar song melodies (e.g., Silent Night), even without accompanying lyrics. It was argued that melodic information may have been processed to some extent and this was enough to co-activate both associative musical information and the lyrics in the speech lexicon, making recognition possible.
}

years) and a mean education level of 15.6 years (range: 11-20 years). Two controls had no musical experience, while the other ten had some experience with singing and/or playing an instrument.

\subsection{Stimuli and materials}

We tested KB on an English version of the music and prosody discrimination tasks used by Patel et al. (1998), constructed and supplied to us by Aniruddh Patel. The stimuli consisted of sentence pairs that differed only in prosody, as well as analogous music pairs that were created by synthesizing a tone sequence that followed the $F_{0}$ and temporal patterns of the syllables in the sentences. There were three types of sentence pairs (see Appendices A-C). (1) There were 12 statement-question pairs that differed in terminal-pitch information (i.e., questions had a rising pitch, while statements had a falling pitch). (2) There were 12 pairs of emphasis-shift pairs that differed in internal-pitch information (i.e., different words carried the emphasis). (3) There were 12 timing-shift pairs that differed in the placement of pauses.

\subsection{Procedure}

\subsubsection{Discrimination tasks}

Prosodic discrimination tasks. There were three prosodic discrimination tasks: terminal-pitch discrimination (i.e., statement-question pairs), internal-pitch discrimination (i.e., emphasis-shift pairs), and rhythm discrimination (i.e., timing-shift pairs).

Each of the 12 statement-question sentences was presented in a same condition (six trials statement/ statement; six trials question/question) and a different condition (six trials statement/question; six trials question/statement), with four randomly chosen sentences being repeated in each of the two conditions, for a total of 32 trials. Each of the 12 emphasis-shift sentences was presented in a similar fashion (i.e., 12 trials same word emphasized; 12 trials different word emphasized, with four randomly chosen sentences being repeated in each of the two conditions). The statement-question and emphasis-shift pairs were presented within in a random interleaved manner across two blocks of 32 trials each (i.e., sentences that differed in pitch accent were presented together). In a separate block of 32 trials, each of the 12 timing-shift sentences was presented in a similar manner to the pitch shift sentences (i.e., 12 trials timing same; 12 trials timing different, with four randomly chosen sentences being repeated in each of the two conditions).

Musical discrimination tasks. The procedure for the musical discrimination tasks was the same as that used for the prosodic discrimination tasks, using the music pair analogues derived from the linguistic stimuli (see Patel et al., 1998). 


\subsubsection{Prosodic perception tasks}

There were three prosodic perception tasks: statement-question categorization, emphasis detection, and syntactic interpretation.

Statement-question categorization. This task tested participants' ability to use prosodic information to categorize a sentence as a statement or a question in the absence of other cues (e.g., lexical). The 12 sentences in Appendix A were randomly presented once as a statement and once as a question, with eight randomly chosen sentences being repeated (four questions; four statements) for a total of 32 trials.

Emphasis detection. This task tested participants' ability to use fundamental frequency information to detect which word in a sentence carried the emphasis. The 12 sentences listed in Appendix B were randomly presented once with the emphasis on one word and once with the emphasis on a different word, with eight randomly chosen sentences being repeated (four word one; four word two) for a total of 32 trials. Participants' were asked to decide from a choice of three alternatives, which word had carried the emphasis. For example, if the sentence was "You sing well, Paul," then participants chose the emphasized word from among: sing, well and Paul.

Syntactic interpretation. This task tested participants' ability to use prosodic information to interpret syntactically ambiguous sentences. Participants' were presented with a question to which only one member of the following timing-shift pair sentences (Appendix C) was the correct answer. Ten of the sentences listed in Appendix $\mathrm{C}$ were randomly presented twice, once with the first alternative being correct and once with the second alternative being correct. Participants' indicated whether the first or second member of the pair was the correct answer. For example, after the question "In which sentence is Henry the child?," the participant heard the phrases "Henry, the little one eats a lot," and "Henry, the little one, eats a lot."

$\mathrm{KB}$ was tested in a quiet, unoccupied room at his nursing home. Due to his health, KB tired easily, thus testing was conducted across several short sessions. KB was tested on the three linguistic discrimination tasks, followed by the musical discrimination tasks, followed by the prosodic perception tasks. Controls were tested in a quiet room at Queen's University. Testing was conducted in one session lasting approximately $2 \mathrm{~h}$, with several short breaks. Controls were tested on the discrimination tasks, followed by the prosodic perception tasks. Half the controls completed the prosodic discrimination tasks first, while the others completed the musical discrimination tasks first. The stimuli were presented on an audiocassette recorder with the volume adjusted to a level that was loud, but not uncomfortable for participants.

\subsection{Results}

On all tasks KB's performance was not significantly better than chance $\left(\chi^{2}\right.$ s $\left.\leqslant 2.00, n s\right)$. His scores fell outside the range of controls on all tasks (see Tables 1 and 2).

\subsubsection{Prosodic and musical discrimination}

Correct responses as a percentage of total trials are reported in Table 1 for both KB and controls. KB was unable to reliably discriminate two stimuli based on terminal-pitch information (i.e., statement-question pairs), internal-pitch information (i.e., emphasis-shift

Table 1

Performance on Prosodic and Musical Discrimination Tasks

\begin{tabular}{|c|c|c|c|c|c|c|}
\hline \multirow[b]{3}{*}{ KB } & \multicolumn{2}{|c|}{ Statement-question pairs } & \multicolumn{2}{|c|}{ Emphasis-shift pairs } & \multicolumn{2}{|c|}{ Timing-shift pairs } \\
\hline & Speech & Music & Speech & Music & Speech & Music \\
\hline & $59.4 \% * * *$ & $62.5 \% * * *$ & $50.0 \% * * *$ & $43.8 \% * *$ & $62.5 \% * *$ & $56.3 \% * *$ \\
\hline \multicolumn{7}{|c|}{ Controls } \\
\hline 1 & $90.6 \%$ & $100 \%$ & $87.5 \%$ & $87.5 \%$ & $93.8 \%$ & $81.3 \%$ \\
\hline 2 & $100 \%$ & $96.9 \%$ & $100 \%$ & $87.5 \%$ & $93.8 \%$ & $68.8 \%$ \\
\hline 3 & $87.5 \%$ & $93.8 \%$ & $90.6 \%$ & $68.8 \%$ & $100 \%$ & $71.4 \%$ \\
\hline 4 & $96.9 \%$ & $93.8 \%$ & $93.8 \%$ & $59.4 \%$ & $93.8 \%$ & $65.6 \%$ \\
\hline 5 & $100 \%$ & $93.8 \%$ & $100 \%$ & $93.8 \%$ & $100 \%$ & $84.4 \%$ \\
\hline 6 & $90.6 \%$ & $93.8 \%$ & $100 \%$ & $96.9 \%$ & $100 \%$ & $96.9 \%$ \\
\hline 7 & $100 \%$ & $84.4 \%$ & $100 \%$ & $81.3 \%$ & $100 \%$ & $93.8 \%$ \\
\hline 8 & $90.6 \%$ & $84.4 \%$ & $84.4 \%$ & $65.6 \%$ & $81.3 \%$ & $65.6 \%$ \\
\hline 9 & $90.6 \%$ & $87.5 \%$ & $100 \%$ & $81.3 \%$ & $100 \%$ & $81.3 \%$ \\
\hline 10 & $90.6 \%$ & $93.8 \%$ & $96.9 \%$ & $87.5 \%$ & $96.9 \%$ & $84.4 \%$ \\
\hline 11 & $100 \%$ & $100 \%$ & $100 \%$ & $100 \%$ & $90.6 \%$ & $90.6 \%$ \\
\hline 12 & $100 \%$ & $100 \%$ & $100 \%$ & $100 \%$ & $93.8 \%$ & $78.1 \%$ \\
\hline Mean & $94.8 \%$ & $93.5 \%$ & $96.1 \%$ & $84.1 \%$ & $91.5 \%$ & $80.2 \%$ \\
\hline$S D$ & $5.1 \%$ & $5.6 \%$ & $5.7 \%$ & $13.5 \%$ & $10.3 \%$ & $10.7 \%$ \\
\hline
\end{tabular}

Note: ${ }^{* * *}$ Three standard deviations below control mean; ${ }^{* *}$ two standard deviations below control mean. Correctly discriminated stimulus-pairs, expressed as the percentage of total trials, for $\mathrm{KB}$ and controls. 
Table 2

Performance on Prosodic Perception Tasks

\begin{tabular}{|c|c|c|c|}
\hline & Statement-question & Emphasis detection & Syntactic interpretation \\
\hline KB & $56.3 \% * * *$ & $46.9 \% * * *$ & $40.0 \% * * *$ \\
\hline \multicolumn{4}{|c|}{ Controls } \\
\hline 1 & $100 \%$ & $96.9 \%$ & $75 \%$ \\
\hline 2 & $100 \%$ & $96.9 \%$ & $95 \%$ \\
\hline 3 & $100 \%$ & $90.6 \%$ & $70 \%$ \\
\hline 4 & $96.9 \%$ & $100 \%$ & $90 \%$ \\
\hline 5 & $100 \%$ & $100 \%$ & $90 \%$ \\
\hline 6 & $93.8 \%$ & $100 \%$ & $75 \%$ \\
\hline 7 & $100 \%$ & $100 \%$ & $90 \%$ \\
\hline 8 & $96.9 \%$ & $93.8 \%$ & $80 \%$ \\
\hline 9 & $100 \%$ & $100 \%$ & $85 \%$ \\
\hline 10 & $100 \%$ & $100 \%$ & $90 \%$ \\
\hline 11 & $100 \%$ & $100 \%$ & $100 \%$ \\
\hline 12 & $100 \%$ & $100 \%$ & $100 \%$ \\
\hline Mean & $99.0 \%$ & $98.2 \%$ & $86.7 \%$ \\
\hline$S D$ & $2.6 \%$ & $3.7 \%$ & $10.4 \%$ \\
\hline
\end{tabular}

Note: ***Three standard deviations below control mean. Correct responses on the three prosodic perception tasks, expressed as the percentage of total trials, for KB and controls.

pairs), or rhythm (i.e., timing-shift pairs) in either the prosodic or musical domains.

Controls were well above chance on both the prosodic and musical discrimination tasks. Paired-sample $t$ tests showed, however, that controls were significantly more accurate at making decisions, using either internalpitch information or timing information, on the prosody tasks than the musical tasks (internal pitch: $t(11)=3.83$, $p<.01$; timing shift: $t(11)=5.71, p<.001)$. Controls' ability make discriminations based on terminal-pitch information did not differ significantly across the prosodic and musical tasks $(t(11)=.66, n s)$.

\subsubsection{Prosodic perception tasks}

Correct responses as a percentage of total trials are reported in Table 2 for both KB and controls. As on the discrimination tasks, KB did not perform significantly above chance on the prosodic perception tasks. His scores were more than three standard deviations below those of controls. He was unable to reliably differentiate statements from questions, detect which word in a sentence carried the emphasis, or interpret syntactically ambiguous sentences based on timing cues.

\section{General discussion}

Segmental speech information and musical information are conveyed by different information in the acoustic signal. Moreover, several lines of evidence indicate that these two types of auditory stimuli are processed by distinct neural mechanisms. In contrast to segmental speech information, prosodic speech information is conveyed by many of the same acoustic cues as certain aspects of music, such as melodic contour and rhythm. In the present investigation we examined the ability of KB to perform both prosodic and musical discrimination tasks, as well as prosodic perception tasks. $\mathrm{KB}$ is an amateur musician who suffers from severe apperceptive amusia consequent to right frontoparietal damage that is quite confined, relative to other cases of amusia. Firstly, we wanted to examine whether KB's deficits on prosodic perception tasks (Nicholson et al., 2002) were related to a deficit in processing acoustic patterns, as appears to be the case for his musical deficits (Steinke et al., 2001). Secondly, we were interested in replicating the finding of Patel and colleagues (1998) that the discrimination performance of both amusic patients and age-matched controls is correlated across the linguistic and musical domains, when pitch and timing parameters are comparable.

The present results confirmed earlier findings that $\mathrm{KB}$ was impaired, relative to age matched controls, on prosodic perception tasks (e.g., deciding whether a sentence was a statement or question; Nicholson et al. (2002)). Further, KB was markedly impaired on discriminating pairs of sentences based on either pitch (terminal and internal) cues or timing cues. This finding indicates that, like his impairment in recognizing familiar music, his impairment in perceiving prosodic categories may be related to a deficit in processing pitch or timing information, rather than a memory deficit.

$\mathrm{KB}$ was also markedly impaired at discriminating pairs of musical phrases derived from the linguistic stimuli, indicating a deficit in processing the acoustic patterns underlying melodic contour and rhythm. His 
performance, however, was at chance on all tasks, making it impossible to correlate his performance across the two domains. ${ }^{3}$ The fact that KB performs at chance on both tasks, as well as the prosodic perception tasks (i.e., which place less demand on memory and attention), suggests that there may be some functional relations across the two domains. This finding indicates that the processing of prosodic and melodic patterns may partially rely on overlapping or adjacent anatomical structures. Of course, an alternative explanation of the findings is that KB impairments may be due to a general deficit in attention, memory or motivation. Although this explanation would be consistent with his performance on tests of frontal lobe function, it is inconsistent with our previous findings (Nicholson et al., 2002). More specifically, we found that KB performs at control levels on segmental auditory speech perception tasks (i.e., identifying words and syllables), indicating that his auditory deficits are specific to musical and prosodic information.

Patel et al. (1998) proposed that a neural system involving the right frontal cortex may be involved in processing prosodic and musical information. More specifically, they suggested that the right frontal cortex might be involved in the short-term retention and comparison of pitch and timing patterns, rather than the extraction of these patterns from the auditory signal (see also Zatorre, 2001). The fact that, like IR, $\mathrm{KB}$ has damage in the right frontal cortex is consistent with this proposal. In the present study, however, we found that KB was impaired on discrimination tasks, as well as prosodic perception tasks, while IR was only impaired on discrimination tasks (Patel et al., 1998). It could be that KB is unable to retain and compare pitch and duration cues even over a single

\footnotetext{
${ }^{3}$ Inconsistent with the findings of Patel et al. (1998), in the present study controls were more accurate on prosodic discriminations, based on internal-pitch and -timing cues, compared to musical discriminations based on the same cues. Controls' performance was not significantly different across the linguistic and musical domains for discriminations based on terminal-pitch cues. The present finding that controls are more accurate at making internal-pitch and -timing discriminations in the speech domain than the musical domain was not due to the poor performance of those two controls without musical experience.

It may be that the prosodic discrimination tasks were easier given that changes in pitch or timing could be associated with certain words, while this is more difficult to do with tones. On the terminal-pitch trials the pitch change could be associated with the last tone, making it less difficult than the internal-pitch and -timing musical discriminations tasks. Also, humans extract pitch and timing information from speech on an everyday basis, but this is not the case for series of tones. Participants were $30-40$ years older in the present study compared to the Patel et al. study. Differences across tasks that may be related to attentional or memory demands might become more apparent with age. Thus, based on control data, the music tasks may have been more difficult than the prosody tasks and required additional neural resources.
}

sentence or musical phrase. It is also possible, however, that KB's deficits arise at a different stage of processing than IR's.

It seems unlikely that KB's deficits arise at the stage of sensory analysis of variations in frequency or duration information in the incoming auditory signal. Recent work suggests that the processing of variations in frequency information is a function of the right primary auditory cortex (for review see Zatorre et al., 2002). The primary auditory cortex is intact bilaterally in KB. In addition, it has been found that individuals (termed congenital amusics) with a deficit in discriminating variations in pitch (Peretz et al., 2002) show a dissociation in performance across the musical and prosodic versions of Patel's tasks (Ayotte, Peretz, \& Hyde, 2002). These individuals were impaired on the musical tasks, but not on the prosodic tasks.

In contrast to IR, KB had extensive damage to the right parietal cortex. Consistent with this, the only other cases of amusia associated with a unilateral lesion have been reported after damage to the right parietal cortex (Griffiths et al., 1997). Griffiths and colleagues found that such patients show a deficit in the localization of sound in space and that this deficit may reflect impairments in the spatial and temporal processing of sound patterns. It may be that KB's impairments on both the musical and prosodic tasks arise at the stage of perceiving pitch or timing patterns in the acoustic stimuli, rather than at the stage of sensory analysis or short-term retention.

In conclusion, the present results in combination with previous findings (Griffiths et al., 1997; Patel et al., 1998) suggest that the right parietal cortex may be involved in the extraction of spatial, pitch and timing patterns from the acoustic signal, while regions in the right frontal lobe may be important for the short-term retention and comparison of such acoustic patterns. Such processes might be necessary for processing some aspects of music as well as speech prosody, but not for processing other auditory stimuli, which do not rely on structured acoustic patterns that unfold relatively slowly over time (i.e., segmental speech information).

\section{Acknowledgments}

We would like to thank KB for his time and patience during testing, A. Patel for providing us with a copy of his tasks, and Justine Dembo and Pamela Seeds for testing the control participants. This research was supported by grants from NSERC, MRC/CIHR, NIH (Grant DC-05774 from the National Institute of Deafness and other Communication Disorders) and the CRL Keihanna Info-Communications Research Laboratories and Telecommunications Advancement Organization of Japan. 


\section{Appendix A. Statement-question pairs}

1. He speaks French./?

2. Francis is at the restaurant./?

3. She drinks three large cups of coffee every morning./?

4. He wants to leave now./?

5. She plays the flute./?

6. He likes to drive fast cars./?

7. He wants to buy a house next to the beach./?

8. She forgot her book./?

9. He has been in Paris for three months./?

10. The supermarket is closed on Sunday./?

11. He works $10 \mathrm{~h}$ a day./?

12. The telephone doesn't work./?

\section{Appendix B. Emphasis-shift pairs}

1. Go in front of the bank, I said.

2. I like blue ties on gentleman.

3. Give me the math exam today.

4. You sing well, Paul.

5. The orange flowers smell very sweet.

6. Take the bus to Boston, Anne.

7. Paul's book is at my house.

8. The pink umbrella is less expensive.

9. He likes to talk romance novels.

10. It's Jack's sister, isn't it?

11. Sing now, please.

12. I need two weeks of vacation.

\section{Appendix C. Timing-shift pairs}

1. Henry, the little one(,) eats a lot.

2. She is named Jenny(,) Sue.

3. Theresa, I love you(,) were his last words.

4. Jack, the athlete(,) runs quickly.

5. Paul, my friend(,) is handsome.

6. Anne, his wife(,) is fat.

7. I am going to see Billie(,) Jean.

8. Madame(,) Flower is the name of my cat.

9. Bob, the chef(,) is going to make our dinner.

10. Francis, the doctor(,) is ready to begin.

11. Say the sentence, "you are handsome" (,) Henry.

12. Tom, my boyfriend(,) is out of town.

\section{References}

Ayotte, J., Peretz, I., \& Hyde, K. (2002). Congenital amusia: a group study of adults afflicted with a music-specific disorder. Brain, 125, 238-251.
Ayotte, J., Peretz, I., Rousseau, I., Bard, C., \& Bojanowski, M. (2000). Patterns of music agnosia associated with middle cerebral artery infarcts. Brain, 123, 1926-1938.

Besson, M., \& Schon, D. (2001). Comparison between language and music. Annals of the New York Academy of Sciences, 930, 232-258.

Blair, J. R., \& Spreen, O. (1989). Predicting premorbid IQ: A revision of the national adult reading test. The Clinical Neuropsychologist, 3, 129-136.

Bolinger, D. (1989). Intonation and its uses: Melody in grammar and discourse. Stanford, CA: Stanford University Press.

Dalla Bella, S., \& Peretz, I. (1999). Music agnosias: Selective impairments of music recognition after brain damage. Journal of New Music Research, 28, 209-216.

Goodglass, H., \& Kaplan, E. (1983). Boston diagnostic aphasia examination (BDAE). Philadelphia, PA: Lee and Febriger. Distributed by Psychological Assessment Resources, Odessa.

Griffiths, T. D. (2002). Central auditory processing disorders. Current Opinion in Neurology, 15, 31-33.

Griffiths, T. D., Rees, A., Witton, C., Cross, P. M., Shakir, R. A., \& Green, G. G. (1997). Spatial and temporal auditory processing deficits following right hemisphere infarction. A psychophysical study. Brain, 120, 785-794.

Heaton, R. K. (1981). A manual for the Wisconsin Card Sorting Test. Odessa, FL: Psychological Assessment Resources.

Nicholson, K. G., Baum, S., Cuddy, L. L., \& Munhall, K. G. (2002). A case of impaired auditory and visual speech prosody perception after right hemisphere damage. Neurocase, 8, 314 322

Patel, A. D., Peretz, I., Tramo, M., \& Labreque, R. (1998). Processing prosodic and musical patterns: A neuropsychological investigation. Brain and Language, 61, 123-144.

Peretz, I. (1996). Can we lose memories for music? The case of music agnosia in a nonmusician. The Journal of Cognitive Neuroscience, $113,1185-1205$.

Peretz, I., Ayotte, J., Zatorre, R. J., Mehler, J., Ahad, P., Penhune, V. B., \& Jutras, B. (2002). Congenital amusia: a disorder of finegrained pitch discrimination. Neuron, 33, 185-191.

Peretz, I., Kolinsky, R., Tramo, M., Labrecque, R., Hublet, C., Demeurisse, G., \& Belleville, S. (1994). Functional dissociations following bilateral lesions of auditory cortex. Brain, 117, 12831301.

Polster, M. R., \& Rose, S. B. (1998). Disorders of auditory processing: Evidence for modularity in audition. Cortex, 34, 47-65.

Raven, J. C. (1965). Guide to using the coloured progressive matrices. London, UK: H.K. Lewis.

Rey, A. (1941). L'examen psychologique dans les cas d'encephalopathy traumatique. Archives de Psychologie, 28, 286-340.

Stone, C. P., Girdner, J., \& Albrecht, R. (1946). An alternate form of the Wechsler Memory Scale. Journal of Psychology, 22, 199-206.

Steinke, W. R., Cuddy, L. L., \& Jakobson, L. S. (2001). Dissociations among functional subsystems governing melody recognition after right hemisphere damage. Cognitive Neuropsychology, 18, 411-437.

Wechsler, D. (1981). Manual for the Wechsler Adult Intelligence Scale (Rev. Ed.). New York, NY: The Psychological Corporation.

Zatorre, R. (2001). Neural specializations for tonal processing. Annals of the New York Academy of Sciences, 930, 193-210.

Zatorre, R., Belin, P., \& Penhune, V. B. (2002). Structure and function of auditory cortex: Music and speech. Trends in Cognitive Neuroscience, 6, 37-46. 